\title{
Permeate Flux in Ultrafiltration Membrane: A Review
}

\author{
A. Beicha ${ }^{1 *}$, R. Zaamouch ${ }^{2}$ \& N. M. Sulaiman ${ }^{3}$ \\ ${ }^{1}$ University of Jijel, Faculty of Sciences and Technology, Department of Process Engineering, PB. 98, Ouled \\ Aissa, 18000 Jijel, Algeria \\ ${ }^{2}$ University of Jijel, Faculty of Sciences and Technology, Department of Mechanics, PB, 98, Ouled Aissa, 18000 \\ Jijel, Algeria \\ ${ }^{3}$ University of Malaya, Chemical Engineering Department, 50603, Kuala Lumpur, Malaysia
}

\begin{abstract}
Membrane processes exist for most of the fluid separations encountered in industry. The most widely used is membrane ultrafiltration, pressure driven process which is capable of separating particles in the approximate size range of 0.001 to $0.1 \mu \mathrm{m}$. The design of membrane separation processes, like all other processes, requires quantitative expressions relating material properties to separation performance. The factors controlling the performance of ultrafiltration are extensively reviewed. There have been a number of seminal approaches in this field. Most have been based on the rate limiting effects of the concentration polarization of the separated particles at the membrane surface. Various rigorous, empirical and intuitive models exist, which have been critically assessed in terms of their predictive capability and applicability. The decision as to which of the membrane filtration models is the most correct in predicting permeation rates is a matter of difficulty and appears to depend on the nature of the dispersion to separated.
\end{abstract}

Keywords: Ultrafiltration, mathematical modelling, permeate flux, limiting flux

\subsection{INTRODUCTION}

Separation and purification processes using membrane technology are gaining popularity in many chemical and food processing as well as in waste treating industries. The technology offers several advantages over and above the traditional techniques, including low energy requirement and low temperature of operation. The ultrafiltration process is found to be suitable for large-scale operations and numerous studies on its commercial application to concentrate or purify solutions and in the extraction of solvents have been reported in the literature.

Ultrafiltration is a pressure driven process for separating particles in the approximate size range of 0.001 to $0.1 \mu \mathrm{m}$. Under a typical hydrostatic

*Correspondting to: A. Beicha (email: abeicha@ yahoo.com) pressure of 60 to $600 \mathrm{kPa}$ applied across the membrane, the solvent is forced through it as permeate. Solutes that are unable to pass through are retained, concentrated and removed tan gential to the membrane surface as retentate. The flow of bulk solution towards the membrane results in concentration polarization, with solute concentration at the membrane surface being higher than that in the bulk. The thickness of this polarized layer is significantly reduced by the magnitude of the cross-flow velocity of the feed solution past the membrane surface, which shears off the layer. Depending on the macromolecules, high osmotic pressure could develop in the polarized layer at the membrane-solution interface which reduces the effective driving force for the flow of permeate. For high molecular weight solute, the effect of osmotic pressure is less important. In certain cases, the solute wall concentration may 
reach its solubility limit and solute precipitation onto the membrane surface to form a gel or a gel layer can occur. Osmotic pressure, concentration polarization and gel layer formation are among the dominant resistances, which have been found to control ultrafiltration and other membrane filtration processes, in addition to membrane fouling by adsorption.

The development of quantitative predictive models is, therefore, of great importance for the successful application of membrane separation processes in the process industries. The design and simulation of membrane separation processes, like all other processes, require quantitative expressions relating material properties to separation performance. The physical theories governing the filtration models principally describe the effect of the concentration polarization phenomena at the membrane surface. This article presents a review of the existing filtration models for colloidal and fine particle dispersions with emphasis on their qualitative and quantitative predictive capability, and their limitations.

\subsection{FACTORS LIMITING PERMEATE FLUX IN ULTRAFILTRATION}

During an actual separation, the membrane performance can change drastically with time, and a typical flux-time behaviour observed which is a decrease in flux through the membrane over time. This flux decline behaviour is mainly due to membrane fouling (deposition of solute at the membrane surface and in pores or phase boundary). The concentration of the accumulated solute molecules over the membrane surface may become so high and exceeds its solubility limit. In this case a gel layer can be formed on the membrane which in turn exerts the gel layer resistance $R_{g}$ This phenomenon mainly happens when the solution contains proteins. It is possible for some solutes to penetrate into the membrane and block the pores thus leading to the pore-blocking resistance $R_{p b}$. Adsorption can also take place on the membrane surface as well as within the pores themselves. Figure 1 provides a schematic representation of the various resistances that can occur.

The extent of these phenomena is strongly dependent on the type of membrane process and feed solution employed [1-5]. Even for a given solution, fouling will depend on physical and chemical parameters such as concentration, temperature, $\mathrm{pH}$, ionic strength and specific interactions such as hydrogen bonding and dipoledipole interaction.

Several parameters may affect the outcome of a deposition process during UF. Persson and Nilsson [6] measured the amount of whey protein deposited, indirectly as deposit resistance, after static protein exposure. They found that the deposit resistance depended on the membrane pore size; the larger the nominal pore size; the less the deposit resistance. Recent experimental investigations [7] suggest that interparticle and especially electrostatic interactions which are important in the colloidal size range of 5-500 nm play an important role. It has been found that the permeability of layers of proteins formed at

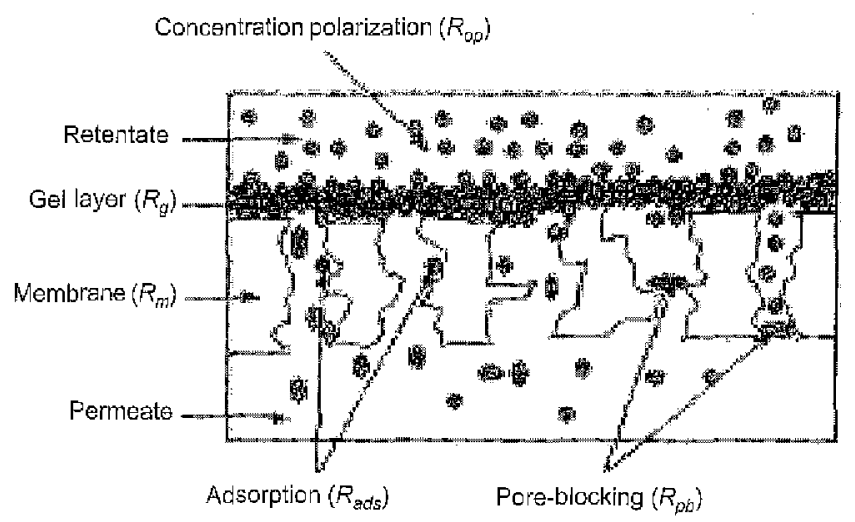

Figure 1 Overview of various resistances to mass transport 
membrane surfaces during ultrafiltration depends on solution conditions [8]. Wakeman and Tarleton [9] have found that varying the $\mathrm{pH}$, and hence the zeta-potential, of anatase dispersions has a fivefold effect on filtration rate. Heinemann et al. [10] studied the effect of $\mathrm{pH}$ and ionic strength on the rejection of whey protein and found that the rejection increased with these two parameters. The increased in rejection was due to electrostatic interaction between solute and membrane.

Fordham and Ladva [11] have studied the crossflow filtration of bentonite suspensions by measuring the filtrate flux with time and post mortem measurements of the steady-state gel thickness. The steady-state flux was observed to increase with increasing cross-flow velocity whereas the gel thickness decreased, indicating that higher shear rates reduced the gel height, and thus lowered the resistance to fluid out flow. Measurements of the filtrate flux, showed that after an initial period of flux decline, a steady-state flux was reached. In general, this steady-state value is found to increase with increasing applied crossflow velocity. However, in a few cases e.g. Fischer and Raasch [12], Lu and Ju [13] and Wakeman and Tarleton [9], reduced values have also been reported which are explained in terms of the selective deposition of fine particles into the filter gel.

Hydrophobicity of particles may affect their deposition on the membrane surface. Persson et al. [14] have studied the fouling behaviour of silica on four different microfiltration membranes. They observed that hydrophobic particles aggregated more and formed a less dense gel layer on the membrane than hydrophilic particles did. They found that heavy fouling on membranes occurred with mixtures of protein with hydrophilic particles but not with hydrophobic particles and protein mixture.

There is considerable experimental evidence that protein adsorption within the pores of ultrafiltration reduces the effective membrane pore size and therefore alter the membrane transport properties [15-18]. The adsorption phenomenon depends on the extent and strength of the interaction governed by the physical and chemical properties of the protein, adsorbent surface and solvent (e.g., pH and ionic strength). Norde et al.
[19] found that protein adsorption is strongly affected by electrostatic interactions, with maximum adsorption attained near the protein isoelectric point, i.e. at the point at which the protein is electrically neutral. The adsorption dependency on $\mathrm{pH}$ is largely determined by the relative contribution of intramolecular hydrophobic interaction to the stabilization of the protein structure in solution [19], Bowen and Gan [20] studied bovine serum albumin (BSA) adsorption on polyvinylidene fluoride membranes, and found that the adsorption was rapid initially, reaching equilibrium after 30 min to $3 \mathrm{hr}$. The adsorption isotherms indicated two different adsorption sites, one of high affinity and the other of low affinity. At $\mathrm{pH}=7$, they did not find any adsorption at all. Exposure of track etched polycarbonate or mica membranes to BSA leads quickly to an irreversible decrease in pore radius roughly comparable to that expected for an adsorbed monolayer $[17,18,21]$. Schultz et al. [22] found little or no change in pore radius with dextran solution but substantial changes with solutions of various proteins. Matthiasson [23] studied the adsorption of BSA in cellulose acetate, polysulphone and polyamide membranes. They found that adsorption was greatest on the hydrophobic polysulphone membranes and was least on the hydrophilic cellulose acetate membranes. Protein adsorption increases with increasing bulk protein concentration and substantially reduces the membrane hydraulic permeability.

Ultrafiltration membranes have an asymmetric structure whereby the hydrodynamic resistance is mainly determined within a small layer of the total membrane thickness [1]. Robertson and Zydney [24] have studied the BSA adsorption in the NOVA and OMEGA polyethersulphone membranes. They have found that even though adsorption in the skin of an asymmetric UF membranes is only a small fraction of the total (skin, substructure, and matrix adsorption), it is this skin adsorption that has such profound effects on the membrane hydraulic permeability as well as membrane transport properties in general.

The effect of hydrodynamic conditions on the adsorption of macromolecules onto the surface were reported by some workers. Fuller and Lee 
[25] reported that hydrodynamic forces can dramatically increase the rate of desorption in polymer systems which are otherwise irreversibly adsorbed under no flow conditions. At the highest velocity gradient and for the higher molecular weights, the film thickness was observed to decrease. Using the membrane hydraulic permeability measurements before and after protein adsorption together with protein adsorption experimental data, Robertson and Zydney [24] indicated that protein adsorption should have substantially different effects on membrane transport characteristics, depending upon the pore size of the membrane. For the larger pore membranes, the pore size is reduced by the size of monolayer adsorption (size of an adsorbed albumin molecule) and for smaller pore membranes, the pores will be blocked partially or completely.

The solvent always plays a double role, affecting both lateral interaction between the adsorbate molecules and determining the effective interaction between the surface and the adsorbate. This means that they adsorb strongly from some solvents, and not from others [26]. The flux decline also depends on temperature and the viscous flow inside the pores. An increase in the temperature by one Kelvin degree decreases the viscosity by $2-3 \%$, such that the flux would in that case, increase correspondingly [27]. A salt addition increased the water permeability by $2-6 \%$, which was explained by electroviscous effects such as reduced streaming potentials and less interference from overlapping electrical double layers [28]. The poor separation efficiency may also be due to wide pore size distribution in the membrane. It may also involve concentration polarization, resistance associated with protein deposition, adsorption, and pore plugging, or a combination of these factors [1].

\subsection{ULTRAFILTRATION MODELS}

\subsection{Gel-polarization Film Model}

In the case of ultrafiltration, the solutes are macromolecules or colloids which tend to form the gel layer on the membrane surface [29]. Solutes are conveyed by permeate flux to the membrane surface, and a portion of them permeate through the membrane, but the rest of them are rejected by the membrane and diffuse back into the bulk solution. At steady state, the quantity of solutes conveyed to the membrane is equal to the sum of those that permeate through the membrane and that which diffuse back.

$$
J \times C_{p}=J \times C-D \frac{d C}{d y}
$$

In the conventional gel-polarization model, the concentration of the gel layer, $C_{g}$ is assumed constant. Thus, boundary conditions at steady state after gel layer formation are:

$$
\left.\begin{array}{l}
y=0 \rightarrow C=C_{b} \\
y=\delta \rightarrow C=C_{g}
\end{array}\right\}
$$

Using these boundary conditions, Eq. (1) is integrated over the boundary layer of thickness $\delta$ over which the concentration varies, to give:

$$
J=k \ln \left(\frac{C_{g}-C_{p}}{C_{b}-C_{p}}\right) ; \quad k=\frac{D}{\delta^{i}}
$$

where $J$ is the flux through the membrane, $C_{p}$ the permeate concentration, $C_{b}$ the bulk stream concentration, $C_{g}$ the gel concentration at the membrane surface, $D$ the diffusivity coefficient, and $k$ the mass transfer coefficient. When the concentration of permeate tends to zero), Eq. (3) becomes:

$$
J=k \ln \left(\frac{C_{g}}{C_{b}}\right)
$$

Equation (4) shows that no solutes are able to pass through the membrane and the flux of solvent (water) is dependent only on the characteristics of $D, C_{g}$ and the boundary layer thickness $\delta$. Equation (4) is ordinarily used for the analysis of ultrafiltration fluxes. Mass transfer coefficient in laminar flow can be evaluated by using the following Lêvéque solution applicable for all thinchannel lengths [30]:

$\mathrm{Sh}=1.62\left(\operatorname{ReSc} \frac{d_{h_{l}}}{L}\right)^{0.33}$ for $100<\operatorname{ReSc} \frac{d_{h}}{L}<5000$ 
where Sh is the Sherwood number, Re the Reynolds number, Sc the Schmidt number, $d_{h}$ the equivalent hydraulic diameter and $L$ the channel length. Hence from Eq. (5), we obtain the mass transfer coefficient:

$$
k=0.816\left(\frac{\dot{\gamma}}{L} D^{2}\right)^{0.33}
$$

where $\dot{\gamma}$ is the fluid shear rate at the membrane surface. For higher power dependence of flux on feed velocity than that indicated by Eq. (5), the correlation of Gröber et al. [31] can be used instead. This correction is applicable for velocity and concentration profiles both developing down the full channel length.

$$
\begin{aligned}
\text { Sh } & =0.664\left(\operatorname{Re} \frac{d_{h_{l}}}{L}\right)^{0.5} \mathrm{Sc}^{0.33} \\
k & =0.664\left(\frac{u_{z}}{L}\right)^{0.5} \frac{D^{0.66}}{v^{0.17}}
\end{aligned}
$$

where $u_{z}$ is the feed velocity and $\mathrm{n}$ the kinematic viscosity. Using values calculated from Eqs. (6) or (8) and the value of $C_{g}$, the concentration at which the flux drops to zero, Eqs. (4) predicts steady-permeate flux less satisfactorily compared with experimental flux [32, 33]. Porter [33] explained that the discrepancy between the predicted values given by Eq. (4) and the data is due in fact to the back-diffusion of particles away from the membrane which is supplemented by a lateral migration of particles due to inertial lift or the so called "tubular-pinch effect". Unfortunately, it was found from hydrodynamic calculations, that the inertial lift velocity is often less than the permeate velocity in typical cross-flow microfiltration systems $[34,35]$. On the other hand, Altena and Belfor [36] showed that for solutions of smaller particles, the permeation drag force dominates compared to inertial force. Shen and Probstein [37] suggest that the discrepancy between Eq. (4) and experimental data is due to the dependence of concentration on diffusion and viscosity. They incorporated the dependence of concentration on $D$ in the steady-state concentration diffusion equation and arrived at an equation similar to Blatt's equation for mass transfer (Eq. (6)) in parallel channel laminar ultrafiltration system, with the coefficient of diffusion calculated at the gel concentration, $C_{F^{\prime}}$ Probstein et al. [38] used this expression of permeate flux in determining the diffusivity and gel concentration in macromolecular solutions. Nakao et al. [39] have analyzed many ultrafiltrate flux data based on this treatment of the gel polarization model. In their analyses, they found that $C_{g}$ determined by the extrapolation on logarithm concentration axis, depended most definitely on the type of apparatus or modules and the experimental conditions. They discovered that a solution, whose concentration was made equal to $C_{g}$ sometimes had fluidity, in sharp contrast to a non-fluid gel-like state, and that the ultrafiltration flux did not become zero when this solution was used as a feed. They were able to measure $C_{g}$ directly and found a relationship between $R_{g}$ and $C_{g}$ of 1.7 power, regardless of the kind of solute used. They were of the opinion that $C_{g}$ has no physical significance.

In order to enhance the accuracy of prediction of limiting flux during filtration of particulate suspensions, a number of mechanisms other then Brownian back diffusion have been proposed. Zydney and Colton [40] proposed that the steadystate permeate flux for cross-flow microfiltration could be predicted by the classical gel-polarization model (Eq. (4)). In place of the Stokes-Einstein particle diffusivity, $D_{0}=\mathrm{KT} / 6 \pi \mu a$, they used a "shear enhanced" particle diffusivity based upon the experiments of Eckstein et al. [41]. A constant diffusion coefficient of $D=0.03 a^{2} \gamma$, where a is particle radius, was used. Due to the approximate expression of shear-diffusivity, and invalidity of Lêvéque solution for mass transfer (Eq. (6)) in dilute solution [42], the expression predicts steady-state permeate fluxes that are an order of magnitude lower than the measured fluxes [35].

Among the arguments against the gel polarization film model are, the gel polarization model itself does not specify what mass transfer coefficient, $k$, should be used, the inability to account for the anomalous low and high flux in colloidal suspensions, and flux dependence on the feed velocity varies with the type of solute and the membrane system [33].

There are other models which proposed to explain the gel formation on the membrane surface 
based on concentration polarization. Le and Howell [43] developed a model for ultrafiltration to predict steady-state permeate flux which is described as the pore-blocking model. They have shown that their model can replace the classical gel-polarization model. Their model postulated that the limiting concentration occurring at the membrane surface as TMP is raised, is a result of the interaction between the solute and the surface. Their model again needs the mass transfer coefficient, $k$, as was in the case of the previous gel-concentration model, limiting concentration and another parameter relating the pore size distribution of membrane to solute particle size.

Trettin and Doshi [42] integrated the concentration diffusion equation in the case of a dead-end system by assuming a concentration profile in the boundary layer and that the solute gel concentration is reached instantaneously at the membrane surface.

Bowen and Jenner [44] have developed a rigorous dynamic mathematical model for predicting the rate of ultrafiltration in dead-end system. The model is based on Darcy's equation and the gel formed was compressible. The local specific resistances are calculated from interparticle interaction approach. The model is only valid for charged colloidal dispersions.

Davis and Leighton [34] have presented a theory which describes the transport of a concentrated layer of particles along a porous wall under laminar flow conditions. A shear induced hydrodynamic diffusion mechanism was proposed to describe the lateral migration of particles away from the porous wall as the layer is sheared. At steady-state, particle diffusion within the layer is balanced by the connective flux of particles toward the porous wall due to the fluid into the wall. Their model predicts the nonlinear velocity and concentration profiles within the sheared particle layer, as well as the layer thickness and the wall concentration.

Romero and Davis [45] have proposed a theoretical model of crossflow microfiltration which includes the time dependent decline of permeate flux due to particle layer build up. The model is based on the shear induced hydrodynamic diffusion mechanism of particle motion within a concentrated flowing layer near the membrane surface, balancing the consecutively driven flux of particles toward the membrane surface.

Another genre of models exists that may be described as particle adhesion models, e.g. the critical flow model of Rautenbach and Schock [46], which assumes that gel deposition ceases when the feed velocity exceeds some multiple of the filtrate flux, the constant of proportionality being determined empirically for a given suspension.

\subsection{Concentration Polarization-osmotic Pressure Model}

These models consider the flux as being limited by the high osmotic pressure arising in the concentration-polarized layer close to the membrane surface, with no gel proposed. The water flux through a membrane of constant permeability is reported by Merten [47]:

$$
J=\frac{\Delta p-\Delta \pi}{\mu R_{m}^{--}}
$$

where $R_{m}$ is the membrane resistance, $\mu$ the solvent viscosity and $\Delta \pi=\pi\left(c_{m}\right)-\pi\left(c_{p}\right)$ with the concentrations $c_{m i}$ and $c_{p}$ at the membrane surface and in the permeate, respectively. The osmotic pressure $\pi$ is often represented in terms of a polynomial

$$
\pi=a_{1} c+a_{2} c^{2}+a_{3} c^{2}
$$

where $a_{1}$ is the coefficient in van't Hoffs law for infinitely dilute solutions and $a_{2}, a_{3}$ represent the non-ideality of the solution.

Goldsmith [48] used Eq. (3) together with Eq. (9) in his analysis, and showed that flux is limited by mass transfer conditions on the feed solution of macromolecules side of the membrane (concentration polarization). Although based on molecular weight considerations, the osmotic pressures of macromolecule solutions would appear to be insignificant.

Brian [49] integrated numerically the convective-diffusion equation at steady-state condition with osmotic pressure controlled permeate flux (Eq. (9)). He incorporated the axial and normal velocities expressions developed firstly by Berman [50] for the Newtonian flow field with normal velocity uniform along the channel length. 
By taking the same profiles of velocities used by Brian and assuming a concentration profile, Leung and Probstein [51] proceeded to integrate the steady-state convection-diffusion equation over the boundary layer. They have compared their integral solution to the finite difference solution. used by Brian and found agreement.

Bowen et al. [52] have followed the same approach taken by Leung and Probstein [51] for predicting the rate of cross-flow membrane ultrafiltration in a rectangular channel with one porous wall. However they focused on a detailed description of the dependence of both osmotic pressure and gradient diffusion coefficient on concentration and physicochemical parameters developed originally to describe the dead-end ultrafiltration of colloids [44].

Vilker et al, [53] solved the unsteady convective diffusion equation in the case of unstirred cell system, together with osmotic pressure-type boundary condition at the membrane surface (Eq. (9)). Regular perturbation theory was used to describe concentration polarization during ultrafiltration of albumin solutions for the case of a highly rejecting membrane. They showed that the flux diminishes like the inverse square root of time.

\subsection{Gel Growth Rate Models}

Many different models have been proposed to predict permeate flux during ultrafiltration and microfiltration. The gel layer and membrane may be considered as two resistances in series, and the permeate flux $u_{p}$; can be calculated from the expression:

$$
u_{p}=\frac{\Delta \mathrm{p}}{R_{I T}+R_{g}}
$$

A gel layer is formed by deposition on the membrane surface and affects the flux as a resistance in series with the membrane resistance. The resistance of the deposit, $R_{g}$ may be expressed as follows:

$$
R_{g}=\tilde{R}_{g} I
$$

where $\widetilde{R}_{g}$ is the specific resistance of deposit, and $l$ the gel layer thickness. The gel growth rate is given by the static gel filtration theory:

$$
\frac{d l}{d t}=\frac{\varepsilon_{b}}{\varepsilon_{g}-\varepsilon_{b}} u_{p}
$$

where $\varepsilon_{b}$ is the solidosity of bulk stream, $\varepsilon_{\dot{g}}$ the solidosity of gel formed, $u_{p}$ the permeate flux, and $t$ the time. This law was established to describe the dynamic ultrafiltration in deadended system. The theory assumes that solidosity of solution over gel is constant and equal to bulk solidosity, $\varepsilon_{b}$, and that the solidosity of gel formed, $\varepsilon_{g}$ is also constant.

Equation (13) indicates that the gel thickness will grow continuously with time resulting in a decrease of permeate flux. Thus the flux in deadended systems is so small as to be virtually non existent. However in cross-flow filtration the situation is different, Porter [33] and others have reported an increase in the permeation flux with increasing tangential shear, indicating that a high shear rate is effective in reducing the gel layer thickness. Some researchers have hypothesized that the gel layer accumulates only until the hydrodynamic shear exerted by the flow of suspension causes the gel to flow tangentially along the membrane surface at a rate. which balances the deposition of particles.

In order to account for the effect of feed velocity on deposition of particles on gel layer, the so called term "function probability" must be incorporated in expression (13), that can be rewritten as follows:

$$
\frac{d l}{d t}=\frac{\varepsilon_{b} u_{p}}{\varepsilon_{g}-\varepsilon_{b}} \gamma
$$

where, $\gamma$ is the fraction of particles transported to membrane surface which achieved deposition. This function, $\gamma$, decreases with time until it reaches the zero value and hence the steady-state is reached. By defining the forces acting on spherical particle transported to the vicinity of the gel suspension interface with a protrusion of finite height, along the direction of the main flow and along the direction of permeation, and by application of principal of moments, Stamatakis and Chi [54] obtained the condition for which the spherical particle remains static and finally arrived at one expression of $\gamma$. The investigators were able to predict the dynamic permeate flux data of Murkes and Carlson [55] on the cross-flow filtration of finely dispersed kaolin at a bulk solidosity of 
0.0033. The unknown gel solidosity and permeability were obtained by curve fitting. By direct experimental observation of particles deposition on the gel surface, Makley and Sherman [56] defined the function, $\gamma$, by measuring the angle between the impacting particle trajectories and the gel surface at which rolling commences. As expected, they also reported the thinning of the gel layer at high cross-flow velocity, but surprisingly observed a continuous decrease in permeate flux with increasing velocity. They indicated that thinner gels produced at higher cross-flow velocities offer more hydratulic resistance than thicker ones formed at lower velocities and related the phenomenon to the packing density of particles in the gel layer. The model contained an adjustable parameter R' (ratio of gel resistance formed at high cross-flow velocity with negligible flux to that with no cross-flow velocity applied) which was fitted to the data. Noticeable variations between predicted and experimental fluxes were observed especially at long operating time.

The common assumption used among investigators to model the process was a constant solidosity of suspension above the gel layer, which was taken to be equal to that of the bulk value. In addition to the feed velocity, the solute concentration in the feed solution would also affect the extent of gel layer formation, a parameter that was not considered in most of the models cited earlier. Furthermore, using the bulk solidosity value to characterize the solution just above the gel layer may only be valid for dilute systems. For concentrated solutions, the development of concentration polarization would result in the solidosity value at the interface to be much higher than the bulk solidosity. Sulaiman et al. [57] and Beicha et al. [58] developed a model which couples the formation of a gel layer on the membrane surface and the presences of a polarized layer above the gel. The model was compared with experimental permeate fluxes obtained from the ultrafiltration of polyethyleneglycol (PEG) using polyethersulfone membrane (9000 MWCO). The model gave an excellent prediction of the permeate fluxes. However, for higher transmembrane pressures the model over predicts the permeate fluxes. The same concept employed by Beicha et al. [58] and Zaamouche et al. [59] was used to predict limiting flux when the permeate flux becomes independent of pressure during tubular ultrafiltration. By combining the effects of the effect of the feed velocity and the bulk concentration, Beicha and Zaamouche [60] showed that the limiting permeate flux can be expressed as:

$$
u_{1 \mathrm{~lm}}=A\left(\varepsilon_{g}-\bar{\varepsilon}_{s t}\right) \sqrt{u_{0}}
$$

where $\tilde{\varepsilon}_{s t}$ is the steady-state value of the average solidosity in the polarized layer, and $u_{0}$ the feed velocity. The constant $A$ depends on the solutemembrane system. The limiting flux was found to be proportional to the square root of the feed velocity. For bulk concentrations near the critical concentration, the concentration polarization has no effect on limiting flux. Its effect is more pronounced when increasing bulk concentration beyond the critical concentration at which the two straight lines on the plot of permeate flux at steadystate versus $\ln$ (bulk concentration) crossed each other [60].

\subsection{Adsorption Models}

Pore restriction models assume that the flux decrease is due to the pore becoming narrower because of adsorption, e.g.:

$$
\frac{J_{1}}{J_{0}}=\frac{\left(r_{0}-\Delta l\right)^{4}}{r_{0}^{4}}
$$

where $J_{1}$ is the pure water flux of fouled membrane, $J_{0}$ the pure water flux of cleaned membrane, $r_{0}$ the pore radius of cleaned membrane, and $\Delta r$ decrease in radius. Equation (16) which is based on the Hagen-Poiseuille equation, indicates that the radius decrease due to adsorbed solute, is assumed to be smeared out equally throughout the pore length and that the pore radius is assumed to be sharply defined.

Simulations have been gradually adopted in virtually any branch of science, due to the gain in time and money they offer compared with practical work. Simulation is especially useful when one considers mechanisms which are kinetically rather than thermodynamically limited.

An alternative way to study the adsorptionpolarization interplay in ultrafiltration is presented 
by Gekas et al. [61]. They incorporated the effects of membrane fouling due to the adsorption of solute to the generalized diffusion equation for the polarized boundary layer in a dead-end ultrafiltration process using plate membranes. This fouling mechanism, which is time dependent, is particularly applicable to the ultrafiltration of proteins. Using the model, these investigators were able to assess individually as well as collectively the roles of concentration polarization and adsorption on the profiles of permeate flux. The effects of cross-flow velocity were only investigated indirectly by varying the values of mass transfer coefficient.

Flora [62] simulated the flux decline due to surface fouling and found good agreement with fouling experiments performed in an unstirred ultrafiltration cell. Doshi. [63] has developed a model for the interplay of adsorption and polarization in an unstirred batch cell. His aim was to identify factors limiting flux in the ultrafiltration of macromolecules.

\subsection{CURRENT \& FUTURE DEVELOPMENTS}

The decision as to which of the filtration models is the most correct in predicting flux values is a matter of difficulty and a certain amount of controversy. Each model appears to be consistent with selected experimental data. A large majority of modeling works on ultrafiltration has been by models based on the concentration-diffusion equation. A good model will lead towards better prediction and optimization of ultrafiltration membrane processes. The resistance models have the advantages of including a description of non-Newtonian polarized layers. A theoretical basis has to be established for the governing model equations to enable further developments.

\section{ACKNOWLEDGEMENT}

The authors are grateful for the financial support from ministry for higher education and scientific research through national programs of research.

\section{REFERENCES}

[1] Mulder, M. 1991. Basic Principles of Membrane Technology. Kluwer Academic Publishers. The Netherlands.

[2] N. Hilal, A. W. Mohammad, B. Atkin and N. Darwish. 2003. Using Atomic Force Microscopy Towards Improvement in Nanoflltration Membrane Properties for Desalination Pretreatment: A Review. Desalination. 157: 137-144.

[3] N. Hilal, H. Al-Zoubi, N. A. Darwish, A. W. Mohammad, M. Abu Arabi. 2004. A Comprehensive Review of Nanofiltration Membranes: Treatment, Pretreatment, Modelling, and Atomic Force Microscopy. Desalination. 170: 281-308.

[4] Beolchini, F. Pagnanelli, F., Michelis, I. D. E. and Veglio, F. 2006. Micellar Enhanced Ultrafiltration for Arsenic (V) Removal: Effect of Main Operating Conditions and Dynamic Modeling. Env. Sci. Tech. 40: 2746 2752.

[5] M. F. A. Goosen, S. S. Sablani, H. Al-Hinai, S. Al-Obeidani, R. Al-Belushi and D. Jackson. 2004. Fouling of Reverse Osmosis and Ultrafiltration Membranes: A Critical Review. Sep.Sci. Tech. 39: 2261-2298.

[6] Persson, K. M. and Nilsson, J. L. 1991. Fouling Resistances Models in MF and UF. Desalination. 80: 123-138.

[7] Riesmeier, B., Kroner, K. H. and Kula, M. R. 1990. Harvest of Microbial Suspensions by Microfiltration, Desalination. 77: 219-33.

[8] Reihanian, H., Robertson, C. R. and Michaels, A. S. 1983. Mechanisms of Polarisation and Fouling of Ultrafiltration Membrane by Proteins. J. Membrane Sci. 16: 237-258.

[9] Wakeman, R. J. and Tarleton, E. S. 1991. Colloidal Fouling of Microfiltration Membranes during the Treatment of Aqueous Feed Streams. Desalination. 83: 35-52.

[10] Heinemann P., Howell J. A. and Bryan R. A. 1988. Microfiltration of Protein Solutions: Effect of Fouling on Rejection. Desalination. 68: 243-250.

[11] Fordham, E. J. and Ladva, H. K. J. 1989. Cross-flow Filtration of Bentonite 
Suspensions, Physico-Chem. Hydrodyn. 11: 411-439.

[12] Fischer, E. and Raasch, J. 1985. Cross-flow Filtration. Ger. Chem. Eng. 8: 211-216.

[13] Lu, W. M. and Ju, S. C. 1989. Selective Particle Deposition in Crossflow Filtration. Sep. Sci. Tech. 24: 517-540.

[14] Persson, K. M., Trägårdh, G. And Dejmek, P. 1993. Fouling Behaviour of Silica on Four Different Microfiltration Membranes. J. Membrane Sci. 76: 51-60.

[15] Wong, H. J. and Quin, J. A. 1976. Hindred Diffusion of Macromolecules in TrackEtched Membranes. Colloid and Interface Sci. 5: 169-178.

[16] Mitchell, B. D. and Deen, W. M. 1986. Effect of Concentration on the Rejection Coefficients of Macromolecules in TrackEtch Membranes. J. Colloid Interface Sci. 113: 132-139.

[17] Jens Haberkamp, Mathias Ernst, Gladys Makdissy, Peter M. Huck and Martin Jekel. 2008. Protein Fouling of Ultrafiltration Membrane: Investigation of Several Factors Relevant for Tertiary Wastewater Treatment. J. Env. Eng. Sci. 7: 651-660.

[18] Heru Susanto and Mathias Ulbricht. 2005. Influence of Ultrafiltration Membrane Characteristics on Adsorptive Fouling with Dextrans. J. Mem. Sci. 266: 132-142.

[19] Norde, W., MacRitchie, F., Nowicka, F. G., and Lyklema, J. 1986. Protein Adsorption at Solid-Liquid Interfaces: Reversibility and Conformation Aspects. J. Colloid Interface Sci. 112: 447-456.

[20] Bowen, W. R. and Gan, Z. 1991. Properties of Microfiltration Membranes: Adsorption of Bovine Serum Albumin at Polyvinylidene Fluoride Membranes. J. Colloid Interface Sci. 144: 254-262.

[21] Deen, W. M. 1987. Hindred Transport of Large Molecules in Liquid-Filled Pores. AIChE J. 33: 1409-1425.

[22] Schultz, J. S., Valentine, R., and Choi, C. Y. 1979. Reflection Coefficients of Homopore Membranes: Effect of Molecular Size and Configuration. J. Gen. Physiol. 73: 49-55.

[23] Matthiasson, E. 1983. The Role of Macromolecular Adsorption in Fouling of
UF Membranes. J. Membrane Sci. 16: 23-36.

[24] Roberison, B. C. and Zydney Z. L. 1990. Protein Adsorption in Asymmetric Ultrafiltration Membranes with Highly Constricted Pores. J. Colloid Interface Sci. 134: 563-573.

[25] Fuller, G. G. and Lee, J.-J. 1984. FlowEnhanced Desorption of Adsorbed Flexible Polymer Chains. In Polymer Adsorption and Dispersion Stability. (Edited by E. D. Goodart and B. Vincent), ACS Symposium Series. 240: 55-64.

[26] Cohen Stuart, M. A., Scheutjens, J. M. H. M. and Fleer, G. J. 1984. The Role of the Solvent in Polymer Adsoption: Displacement and Solvency Effects. In Polymer Adsorption and Dispersion Stability (Edited by E. D. Goodart and B. Vincent), ACS Symposium Series. 240: 145-156.

[27] Vigneswaran, S. and Kiat Wong Yen. 1988. Detailed Investigation of Effects of Operating Parameters of Ultrafiltration using a Laboratory-scale Ultrafiltration Unit. Desalination. 70: 299-316.

[28] Persson, K. M. 1994. Characterization and Fouling Studies of MF and UF Membranes. Doctoral Dissertation, Dep. Food Eng., Lund University. Sweden.

[29] Michaels, A. S. 1968. New Separation Technique for the CPI. Chem. Eng, Prog. 64: 31-43.

[30] Blatt, W. F., Dravid, A., Michaels, A. S., Nelsen, L. 1970. In Membrane Science and Technology. J, E. Flinn. (ed.). New York: Plenum Press.

[31] Gröber, H., Erk, S., Grigull, U. 1961. Fundamentals of Heat Transfer. New York: McGraw- Hill.

[32] Grieves, R. B., Bhattacharyya, D., Schomp, W. G., and Bewley, J. L. 1973. Membrane Ultrafiltration of a Nonionic Surfactant. AIChEJ. 19:766-773.

[33] Porter, M. C. 1972. Concentration Polarization with Membrane Ultrafiltration. Ind. Eng. Chem. Prod. Res. Develop. 11: 234-248.

[34] Davis, R. H. and Leighton, D. T. 1987. Shearinduced Transport of a Concentrated Particle 
Layer Along a Porous Wall. Chem. Eng. Sci. 42: 275-281.

[35] Romero, C. A. and Davis, R. D. 1991. Experimental Verification of the Shearinduced Hydrodynamic Diffusion Model of Crossflow Microfiltration. J. Membrane Sci, 62: 249-273.

[36] Altena, F. W. and Belfor, G. 1984. Lateral Migration of Spherical Particles in Porous Flow Channels: Application to Membrane Filtration. Chem. Eng. Sci. 39: 343-355.

[37] Shen, J. J. S. and Probsteine, R. F. 1977. On the Prediction of Limiting Flux in Laminar Ultrafiltration of Macromolecular Solution. Ind. Eng. Chem. Fund. 16: 459-465.

[38] Probsteine, R. F., Leung, W. F. and Alliance, Y. 1979. Determination of Diffusivity and Gel Concentration in Macromolecular Solutions by Ultrafiltration. J. Phys. Chem. 83: $1228-123$.

[39] Nakao, S. I., Nomura, T. and Kimura, S. 1979. Characteristics of Macromolecular Gel Layer Formed on Ultrafiltration Tubular Membrane. AIChE J. 25: 615-62.

[40] Zydney, A. L., and Colton, C. K. 1986. A Concentration Polarization Model for the Filtrate Flux in Cross-Flow Microfiltration of Particulate Suspensions. Chem. Eng. Comm. 47: 1-8.

[41] Eckstein, E. C., Bailay, D. G. and Shapiro, A. H. 1977. Self-diffusion of Particles in Shear Flow of a Suspension. J. Fluid Mech. 79: 191208.

[42] Trettin, D. R. and Doshi, M. R. 1980. Ultrafiltration in an Unstirred Batch cell. Ind. Eng. Chem. Fund. 19: 189-194.

[43] Le, M. S. and Howell, J. A. 1984. Alternative Model for Ultrafiltration. Chem. Eng. Res. Dev. 62: 373-380.

[44] Bowen, W. R. and Jenner, F. 1995. Dynamic Ultrafiltration Model for Charged Colloidal Dispersions: A Wigner-Seitz Cell Approach. Chem. Eng. Sct. 50: 1707-1736.

[45] Romero, C. A. and Davis, R. H. 1990. Transient Model of Crossflow Microfiltration. Chem. Eng. Sci. 45: 13-25.

[46] Rautenbach, R. And Schock, G, 1988. Ultrafiltration of Macromolecular Solutions and Cross-flow Microfiltration of Colloids
Suspensions: A Contribution to Permeate Flux Calculations. J. Membrane Sci. 36: 231-24.

[47] Merten, U. 1963. Flow Relationships in Reverse Ssmosis. Ind. Eng. Chem. Fund. 2: 229-23.

[48] Goldsmith, R. L. 1971. Macromolecular Ultrafiltration with Microporous Membranes. Ind. Eng. Chem. Fund, 10: 113-120.

[49] Brian, P. L. T. 1965. Concentration Polarization in Reverse Osmosis Desalination with Variable Flux and Incomplete Salt Rejection. Ind. Eng. Chem. Fund 4: 439-445.

[50] Berman, A. S. 1953. Laminar Flow in Channels with Porous Walls. J. Applied Physics. 24: 1232-1235.

[51] Leung, W. F., Probstein, R. F. 1979. Low Polarization in Laminar Ultrafiltration of Macromolecular Solutions. Ind. Eng. Chem. Fund. 18: 274-277.

[52] Bowen, W. R., Mongruel, A., and Williams, P. M. 1996. Prediction of the Rate of Crossflow Membrane Ultrafiltration: A Colloidal Interaction Approach. Chem. Eng. Sci. 51: 4321-4333.

[53] Vilker, V. L., Colton, C. K., Smith, K. A. 1981. Concentration Polarization in Protein Ultrafiltration. AIChE J. 27: 632-645.

[54] Stamatakis, K. And Tien Chi. 1993. A Simple Model of Cross-Flow Filtration Based on Particle Adhesion. AIChE J. 39: 12921302.

[55] Murkers, J. and Carlson, C. G. 1988. Cross Flow Filtration: Theory and Practice. New York: John Wiley \& Sons.

[56] Mackley M. R. and Sherman, N. E. 1992. Cross-Flow Gel Filtration Mechanisms and Kinetics. Chem. Eng. Sci. 47: 30673084.

[57] M. Z. Sulaiman, N. M. Sulaiman and A. Beicha, 2001. Prediction of Dynamic Permeate Flux During Cross-flow Ultrafiltration of Polyethylene Glycol using Concentration Polarization-gel Layer Model. J. Mem. Sci. 189: 151.

[58] A. Beicha, R. Zaamouche and N. M. Sulaiman. 2009. Dynamic Ultrafiltration Model Based on Concentration Polarization- 
Gel Layer Interplay. Desalination. 242: 138.

[59] R. Zaamouche, A. Beicha and N. M. Sulaiman. 2009. Cross-flow Ultrafiltration Model Based on Concentration Polarization, J. Chem. Eng. Japan. 42: 107.

[60] A. Beicha and R. Zaamouche. 2009. Limiting Flux Prediction during Tubular Ultrafiltation. Chem. Eng. J. 153:231-235.

[61] Gekas, V., Aimar, P., Lafaille, J. and Sanchez, V. 1993. A Simulation Study of the
Adsorption-concentration Polarization Interplay in Protein Ultrafiltration. Chem. Eng. Sci. 48: 2753-2765.

[62] Flora, J. R. V. 1993. Stochastic Approach to Modeling Surface Fouling of Ultrafiltration Membrane. J. Membrane Sci, 76: 85-88.

[63] Doshi, M. R. 1986. Limiting Flux in the Ultrafiltration of Macromolecular Solutions. in Reverse Osmosis \& Ultrafiltration (Sourirajan S. and Matsuura T. Edition), ACS Symposium Series. 281: 209-223. 